\title{
Urinary tract infection diagnosis and response to therapy in long-term care: A prospective observational study
}

\author{
Peter Daley MD MSc FRCPC DTM+H ${ }^{1}$, Carla Penney BSc ${ }^{1}$, Susan Wakeham BSc ${ }^{1}$, Glenda Compton MN RN², \\ Aaron McKim MD CCFP², Judy O'Keefe MSW RSW ${ }^{2}$, Brendan Barrett MD FRCPC ${ }^{1}$, Lindsay Nicolle MD FRCPC ${ }^{3}$
}

\begin{abstract}
P Daley, C Penney, S Wakeham, et al. Urinary tract infection diagnosis and response to therapy in long-term care: A prospective observational study. Can J Infect Dis Med Microbiol 2015;26(3):133-136.
\end{abstract}

BACKGROUND: The prevalence of asymptomatic bacteriuria among residents of long-term care (LTC) facilities is high, and is a source of inappropriate antibiotic prescription.

OBJECTIVE: To establish symptoms and signs associated with a positive urine culture, and to determine whether antibiotic therapy is associated with functional improvement.

METHODS: A total of 101 LTC patients were prospectively observed after submission of urine for culture.

RESULTS: The culture positivity rate was consistent with the expected asymptomatic bacteriuria rate. Change in mental status and male sex were associated with culture positivity. Treatment decisions were not consistent with culture results. Treatment did not lead to improvement in activities of daily living scores at two days or seven days.

DISCUSSION: Significant growth cannot be well predicted based on clinical variables; thus, the decision to submit urine is somewhat arbitrary. Because urine culture testing and treatment does not lead to functional improvement, restricting access to the test may be reasonable.

CONCLUSION: Urine culture testing in LTC facilities does not lead to functional improvement.

Key Words: Antibiotic stewardship; Asymptomatic bacteriuria; Diagnosis; Long-term care; Urinary tract infection

$\mathrm{U}$ rinary tract infection (UTI) is a common diagnosis among residents in long-term care (LTC) settings, and a common reason to prescribe antibiotics. The annual prevalence of antibiotic use in LTC is high - between $47 \%$ and $79 \%$ (1), or $5.9 \%$ of all residents on a given day (2). The rate of antibiotic use without adequate clinical rationale has been reported to be as high as 38\% in 42 facilities (3) and $42 \%$ in one facility (4).

Attention to antimicrobial stewardship through appropriate antibiotic use can reduce drug costs (5), and can reduce Clostridium difficile diarrhea rates in LTC (6). For these reasons, LTC is an appropriate population in which to study antibiotic stewardship and to intervene to improve use. A cluster randomized study has shown that implementing consensus treatment guidelines for UTI in LTC can reduce antibiotic consumption for UTI (7).

Residents of LTC facilities without indwelling urinary catheters have a prevalence of asymptomatic bacteriuria ( $>10^{8}$ colony-forming units/L)
Le diagnostic d'infection urinaire et la réponse au traitement en soins de longue durée : une étude d'observation prospective

HISTORIQUE : La prévalence de bactériuries asymptomatiques est élevée chez les résidents d'établissements de soins de longue durée (SLD). Elle suscite la prescription inappropriée d'antibiotiques.

OBJECTIF : Déterminer les signes et symptômes associés à une culture d'urine positive et établir si l'antibiothérapie favorise une amélioration fonctionnelle.

MÉTHODOLOGIE : Au total, 101 patients en SLD ont fait l'objet d'une observation prospective après l'envoi d'un prélèvement d'urine pour culture.

RÉSULTATS : Le taux de cultures positives était conforme au taux prévu de bactériuries asymptomatiques. La détérioration de l'état mental et le sexe masculin s'associaient à des cultures positives. Les décisions thérapeutiques n'étaient pas en accord avec les résultats des cultures. Le traitement ne suscitait pas d'amélioration à l'indice d'activités de la vie quotidienne au bout de deux ou sept jours.

EXPOSÉ : Les variables cliniques ne permettent pas de prévoir une croissance importante. Ainsi, la décision de faire une culture d'urine est quelque peu arbitraire. Puisque les cultures d'urine et le traitement n'assurent pas d'amélioration fonctionnelle, il est peut-être raisonnable de restreindre l'accès aux analyses.

CONCLUSION : Dans les établissements de SLD, les analyses d'urine ne favorisent pas d'amélioration fonctionnelle.

of approximately $40 \%$ (8). Screening for or treatment of asymptomatic bacteriuria is not associated with reduction in mortality (8-10), and may contribute to inappropriate antibiotic use, which drives the selection of antibiotic resistance among bacteria in the patient's resident flora (11).

Among LTC residents with a change in clinical status, positive urine culture is often interpreted as the cause, leading to overdiagnosis of UTI. In a recent prospective study involving 399 clinically suspected UTI episodes, there was very poor correlation among symptoms, urine culture results and the decision to use antibiotics (12). A multivariate logistic regression for factors associated with bacteriuria and pyuria revealed that dysuria, change in character of urine and change in mental status were statistically associated with bacteriuria and pyuria, although associations were weak (RR 1.38 to 1.58). Clearly, the diagnosis and treatment of UTI in LTC is not simple or consistent, and evidence to guide the decision to collect urine culture is not available.

${ }^{1}$ Memorial University; ${ }^{2}$ Long-Term Care, Eastern Health, St John's, Newfoundland and Labrador; ${ }^{3}$ University of Manitoba, Winnipeg, Manitoba Correspondence: Dr Peter Daley, Room 1J421, Health Sciences Center, 300 Prince Phillip Drive, Saint John's, Newfoundland and Labrador A1B 3V6. Telephone 709-777-2089, e-mail pkd336@mun.ca 


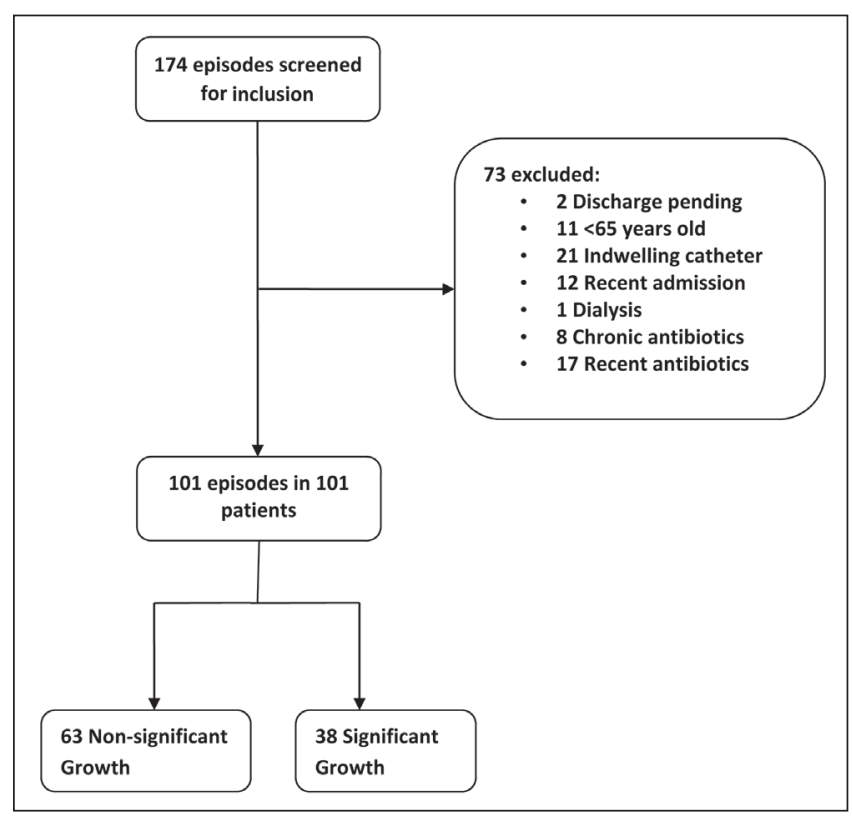

Figure 1) Participant recruitment

Confounding the assessment of the relationship between these three clinical variables and positive laboratory results is the fact that clinical decline, for various reasons, can lead to dehydration, causing a change in character of urine and change in mental status. These signs are often considered to be the result of a UTI (rather than dehydration) and treated as such. Residents with cognitive impairment are more likely to have positive urine cultures (8) and are also more vulnerable to delirium, which frequently leads clinicians to interpret a causal relationship between changes in mentation and bacteriuria. To date, no study has considered the clinical outcome after antibiotic therapy to validate the diagnostic criteria. We performed a prospective observational efficacy study to identify symptoms associated with a positive urine culture, and assessed clinical response to antibiotic therapy.

\section{METHODS}

Six LTC facilities in St John's, Newfoundland and Labrador, ranging from 40 beds to 377 beds, were selected for inclusion based on their high urine culture submission rates. Consecutive urine samples received in the central microbiology laboratory from these LTC facilities between June 24 and July 17, 2013 and between January 20 and March 20, 2014 were identified, and a research associate visited the relevant facility on the day of submission. Receipt of urine for culture was considered to be evidence that nursing staff suspected UTI. Exclusion criteria were applied as follows:

- Not anticipated to remain in the nursing home for LTC (ie, shortterm residents or pending discharge);

- Anticipated life expectancy $<4$ weeks as assessed by nursing staff;

- $<65$ years of age;

- Indwelling catheter or surgical urinary collection device;

- Current renal dialysis;

- Current anti-infective therapy for any reason;

- Resided in the nursing home for $<4$ weeks (providers did not have adequate time to determine resident's baseline functional status).

The study was approved by local health research ethics authority to approach nursing staff for consent to participate. Consent forms and study information sheets were circulated to LTC facilities before the study. The nurse (or delegate) who collected the urine specimen was identified and interviewed on the day of collection, and again after two days and five to seven days. Patient information, including
TABLE 1

Demographic characteristics

\begin{tabular}{lc}
\hline Characteristic $(\mathbf{n}=\mathbf{1 0 1})$ & \\
\hline Age, years, mean \pm SD & $84.0 \pm 8.6$ \\
Female sex, \% & 79.2 \\
Number of comorbidities, mean \pm SD & $1.8 \pm 1.0$ \\
Dementia & $58(57.4)$ \\
Stroke & $13(12.9)$ \\
Liver disease & $1(1.0)$ \\
Depression & $41(40.6)$ \\
Kidney disease & $12(11.9)$ \\
Diabetes & $25(24.8)$ \\
Cancer & $9(8.9)$ \\
Chronic obstructive pulmonary disease & $15(14.9)$ \\
Baseline ADL score, mean \pm SD (independent $=0$, & $11.9 \pm 8.7$ \\
total dependence $=28)$ & \\
Mental status in past seven days & \\
Disorganized speech & $12(11.9)$ \\
Altered perception & $19(18.8)$ \\
Unresponsiveness & $1(1.0)$ \\
Lethargy & $19(18.8)$ \\
\hline Data presented as $n$ (\%) unless otherwise indicated. & ADL Activities of daily \\
living
\end{tabular}

comorbidity and baseline functional capacity (28-point activities of daily living $[\mathrm{ADL}]$ scale including mobility in bed, transfers, locomotion, dressing, toileting, personal hygiene and feeding, and fourpoint mental status scale [12]), was collected from the nurse. The patient, the family, the caregivers and the attending physician were not interviewed. Urine culture was performed quantitatively, according to laboratory protocol.

Univariate logistic regression was performed using SPSS version 20.0 (IBM Corporation, USA) to determine whether nurse-reported clinical symptoms were associated with the primary outcome: growth of at least $10^{8}$ colony-forming units/L of uropathogenic bacteria in urine culture. Symptoms and functional status were described over time. Change in ADL score over time was analyzed using a pairedsamples $t$ test (two-sided).

Variable(s) considered to be potentially predictive of significant growth included demographic characteristics (age, sex, LTC facility, total comorbidities), reasons for collection (change in mental status, change in behaviour, change in character of urine, fever, change in gait or fall, change in voiding pattern, flank pain, patient or family request, abnormal laboratory test result, dysuria, change in functional status, previous UTI, malaise), baseline functional capabilities (ADL score, disorganized speech in the past seven days, altered perception in the past seven days, unresponsive episodes in the past seven days and lethargy in the past seven days), and nurse-reported symptoms (fever, change in behaviour, change in mental status, diarrhea, abdominal pain, shortness of breath, weakness, dysuria, change in character of urine, change in frequency of urination and flank pain). Variables with $\leq 2$ positive responses were removed (change in functional status, change in gait or fall, results of other workup performed, other concurrent infection, dehydration, shortness of breath, syncope, diarrhea, baseline unresponsiveness, cough).

\section{RESULTS}

A total of 174 urine specimens were considered, and 101 episodes from 101 patients were observed (Figure 1). Thirty-eight (37.6\%) of 101 episodes demonstrated significant growth of bacteria in urine. In $36.6 \%$ of episodes, the nurse making the decision to collect urine was interviewed; in other episodes, alternate nurses also familiar with the patient were interviewed. Patient demographics are summarized in Table 1. The mean $( \pm$ SD) age was $84.0 \pm 8.6$ years, $79.2 \%$ were women and there were a mean of $1.8 \pm 1.0$ comorbidities per patient. Baseline 
TABLE 2

Reasons for urine culture collection $(n=101)$

\begin{tabular}{lc}
\hline Clinical reason & $\mathbf{n}(\%)$ \\
\hline Change in behaviour & $35(34.7)$ \\
Dysuria & $31(30.7)$ \\
Change in character of urine & $30(29.7)$ \\
Change in mental status & $26(25.7)$ \\
Change in voiding pattern & $21(20.8)$ \\
Other reason & $14(13.9)$ \\
Patient or family request & $11(10.9)$ \\
Previous urinary tract infection & $11(10.9)$ \\
Flank pain & $8(7.9)$ \\
Malaise & $6(5.9)$ \\
Fever or chills & $5(5.0)$ \\
Abnormal laboratory test result & $4(4.0)$ \\
Change in gait or fall & $3(3.0)$ \\
Change in functional status & $1(1.0)$ \\
\hline
\end{tabular}

ADL score was $11.9 \pm 8.7$, with zero representing total independence and 28 representing total dependence.

Predefined reasons for urine collection ranged from one to seven reasons (mean 2.0 reasons), and are listed in descending order of frequency in Table 2 . The previously described significant clinical predictors (dysuria, change in character of urine and change in mental status) were among the most common reasons. Other reasons for collection included pyuria ( $n=4$ patients), hyperglycemia $(n=2)$, test of cure $(n=1)$, urine retention $(n=1)$, hematuria $(n=1)$, "routine checkup" $(\mathrm{n}=1)$, abdominal pain $(\mathrm{n}=1)$, previous sample mixed growth $(n=1)$ and doctor's request $(n=1)$.

Most episodes did not have adequate record of vital signs or blood testing performed to apply published diagnostic criteria. Body temperature was measured for eight $(7.9 \%)$ episodes, blood pressure was measured for six $(5.9 \%)$ episodes, a dipstick test was performed for $25(24.8 \%)$ episodes and a complete blood count was obtained for $16(15.8 \%)$ episodes. Among 38 urine specimens with significant growth, 25 (65.8\%) grew Escherichia coli, five (10.5\%) grew Proteus species, four (13.2\%) grew Klebsiella species, three $(7.9 \%)$ grew Enterococcus species and one (2.6\%) grew yeast.

Using univariate regression, there were two predictors that were significantly associated with the outcome (male sex, RR 5.58 [95\% CI 1.23 to 25.43]; and change in mental status, RR 13.83 [95\% CI 1.81 to 105.81]). Change in character of urine approached significance (RR 14.51 [95\% CI 0.66 to 320.71]) (Table 3).

Antibiotic treatment was given in 48 of 101 (47.5\%) episodes (Table 4). Treatment decision did not correlate well with significant growth (kappa $=0.44)$. Nineteen $(40 \%)$ of 48 of antibiotic prescriptions were given to patients without significant growth, and nine $(17.0 \%)$ of 53 episodes with significant growth were not treated. Using univariate regression, no clinical or demographic variables were associated with the decision to treat. Significant growth was predictive of the decision to treat (RR 40.01 [95\% CI 4.00 to 401.53]). Mean time between collection and treatment was 1.44 days (range eight days before collection to 10 days after collection). In 21 (43.8\%) of 48 cases treated, treatment was given before preliminary culture results were provided. Among episodes with significant growth that were treated, four $(14.3 \%)$ of 28 patients were given an antibiotic to which the bacteria was reported to be resistant.

ADL score at baseline (before decompensation prompting urine collection) was compared with ADL score at $48 \mathrm{~h}$ (the time period required for culture results to become available), and ADL score at five days (resolution of episode), in groups based on significant growth and treatment (Table 5). There was no significant difference observed in any group between baseline and $48 \mathrm{~h}$, or between $48 \mathrm{~h}$ and five to seven days.
TABLE 3

Univariate correlation of predictors with significant growth ( $n=38$ of 101 episodes)

\begin{tabular}{|c|c|c|c|c|}
\hline Characteristic & $\mathbf{n}$ & RR & $95 \% \mathrm{Cl}$ & $\mathbf{P}$ \\
\hline Age & 101 & 0.99 & $0.93-1.06$ & 0.79 \\
\hline Male sex & 21 & 5.58 & $1.23-25.43$ & 0.026 \\
\hline Total comorbidities & 101 & 0.87 & $0.53-1.47$ & 0.61 \\
\hline Long-term care facility & 101 & 1.39 & $0.95-2.02$ & 0.087 \\
\hline \multicolumn{5}{|l|}{ Stated reason for urine collection } \\
\hline Fever & 5 & 0.00 & & 1.0 \\
\hline Change in voiding pattern & 21 & 16.70 & $0.16-1754.36$ & 0.24 \\
\hline Patient or family request & 11 & 0.12 & $0.007-1.91$ & 0.13 \\
\hline Abnormal laboratory test result & 4 & 2.85 & $0.16-51.27$ & 0.48 \\
\hline Previous urinary tract infection & 11 & 1.28 & $0.19-8.48$ & 0.80 \\
\hline \multicolumn{5}{|l|}{ Baseline functional capacity } \\
\hline Baseline ADL score & 101 & 1.03 & $0.97-1.10$ & 0.38 \\
\hline Baseline disorganized speech & 12 & 1.02 & $0.14-7.59$ & 0.97 \\
\hline Baseline altered perception & 19 & 0.36 & $0.05-2.60$ & 0.31 \\
\hline Baseline periods of lethargy & 19 & 4.20 & $0.91-19.37$ & 0.065 \\
\hline \multicolumn{5}{|l|}{ New symptoms } \\
\hline Change in behaviour & 32 & 3.41 & $0.11-104.90$ & 0.48 \\
\hline Change in mental status & 37 & 13.83 & $1.81-105.81$ & 0.011 \\
\hline Abdominal pain & 10 & 0.68 & $0.083-5.60$ & 0.72 \\
\hline Weakness & 10 & 0.90 & $0.026-30.91$ & 0.95 \\
\hline Worsening in ADL score & 4 & 0.00 & & 1.0 \\
\hline Dysuria & 32 & 2.10 & $0.14-32.13$ & 0.60 \\
\hline Change in character of urine & 30 & 14.51 & $0.66-320.71$ & 0.090 \\
\hline Flank pain & 9 & infinity & & 1.0 \\
\hline Change in frequency of urination & 23 & 0.079 & $0.002-2.62$ & 0.16 \\
\hline
\end{tabular}

ADL Activities of daily living

\section{TABLE 4}

Treatment decision

\begin{tabular}{lccc}
\hline & \multicolumn{2}{c}{ Growth } & \\
\cline { 2 - 3 } Treatment decision & Significant & Nonsignificant & Total \\
\hline Antibiotic prescribed & $29(60)$ & $19(40)^{*}$ & 48 \\
Antibiotic not prescribed & $9(17)^{*}$ & $44(83)$ & 53 \\
Total & 38 & 63 & 101
\end{tabular}

Data presented as $n$ or $n(\%)$. *Inappropriate treatment decision

\section{DISCUSSION}

The objective of the present study was to identify the symptoms or signs associated with the nurse's (or delegate's) decision to submit urine for culture, and to assess the clinical response to antibiotic therapy. We found two predictors of significant growth (male sex and change in mental status). Most examined clinical indicators were not significantly predictive of positive culture. A sex difference may have been related to a cleaner urine collection among males, leading to less mixed growth, or reflect patterns of urine collection among nurses.

Our study had significant limitations. Because of the small sample size, CIs for RRs were very wide. We considered input from nurses only, and family or physicians may have had different perceptions of clinical symptoms and signs. However, nurses in LTC make the decision to collect urine specimens. We were not able to interview the nurse who collected the specimen in many cases because that individual had finished the work shift. Nurses working on the next shift may not have completely understood the reasons for urine collection.

Our study demonstrates that the application of surveillance diagnostic criteria developed for LTC may be difficult, due to the lack of patient investigation performed in the LTC setting.

Consistent with previous studies (13), the observed rate of significant growth among patients with urine collected (38\%) closely matches published rates of asymptomatic bacteriuria in this population 
TABLE 5

Change in activities of daily living (ADL) score*

\begin{tabular}{|c|c|c|c|c|c|c|}
\hline Group & $\mathbf{n}$ & $\begin{array}{c}\text { Mean ADL, } \\
\text { baseline }\end{array}$ & $\begin{array}{c}\text { Mean ADL, } \\
48 \mathrm{~h}\end{array}$ & $\begin{array}{c}\mathrm{P} \text {, baseline } \\
\text { to } 48 \mathrm{~h}\end{array}$ & $\begin{array}{c}\text { Mean ADL, } \\
\text { 5-7 days }\end{array}$ & $\begin{array}{l}P, 48 \mathrm{~h} \text { to } \\
5-7 \text { days }\end{array}$ \\
\hline Culture positive, treated & 28 & 11.5 & 12.2 & 0.30 & 12.2 & No difference \\
\hline Culture positive, not treated & 9 & 17.6 & 16.4 & 0.35 & 16.4 & No difference \\
\hline Culture negative, treated & 18 & 12.1 & 12.1 & No difference & 12.1 & No difference \\
\hline Culture negative, not treated & 44 & 10.9 & 11.2 & 0.29 & 11.3 & 0.34 \\
\hline
\end{tabular}

*Zero represents total independence, 28 represents total dependence

(40\% [8]), indicating that clinical suspicion has no predictive capacity. Pretest probability in the symptomatic patient equals pretest probability in the asymptomatic patient, meaning the clinical suspicion of the diagnosis of UTI is not contributory. The present study did not include patients with indwelling catheters, among whom the rate of bacteriuria would be expected to be higher.

Urine culture results did not influence physician prescription decisions because physicians chose to treat culture-negative patients, to not treat culture-positive patients, to treat before culture results, and to use antibiotics to which the laboratory reported the organisms to be resistant.

Furthermore, we observed no functional improvement from antibiotic treatment. ADL scores did not change over two days or seven days in any treatment group. Using this functional status outcome, neither the test result nor the therapy led to clinical benefit. Without a randomized design, culture-positive and culture-negative patients may have been different before the study began; thus, this estimate may be biased. Culturepositive patients who were not treated had worse ADL scores at baseline, suggesting that lower functioning patients may be selected for no treatment because of a poor prognosis. Although predictors for significant growth may be defined by observational studies, if treatment of significant growth does not lead to clinical improvement, then significant growth is not a suitable outcome. Further research should define UTI diagnostic criteria based on clinical benefit following successful treatment.

\section{REFERENCES}

1. van Buul LW, van der Steen JT, Veenhuizen RB, et al. Antibiotic use and resistance in long term care facilities. J Am Med Dir Assoc 2012;13:568.e1-13.

2. Daneman N, Gruneir A, Newman A, et al. Antibiotic use in long-term care facilities. J Antimicrobial Chemother 2011;66:2856-63.

3. Zimmer JG, Bentley DW, Valenti WM, Watson NM. Systemic antibiotic use in nursing homes. A quality assessment. J Am Geriatr Soc 1986;34:703-10.

4. Peron EP, Hirsch AA, Jury LA, Jump RL, Donskey CJ. Another setting for stewardship: High rate of unnecessary antimicrobial use in a veterans affairs long-term care facility. J Am Geriatr Soc 2013;61:289-90.

5. Goff DA. Antimicrobial stewardship: Bridging the gap between quality care and cost. Curr Opin Infect Dis 2011;24(Suppl 1):S11-20.

6. Brakovich B, Bonham E, Vanbrackle L. War on the spore: Clostridium difficile disease among patients in a long-term acute care hospital. J Healthc Qual 2013;35:15-21

7. Loeb M, Brazil K, Lohfeld L, et al. Effect of a multifaceted intervention on number of antimicrobial prescriptions for suspected urinary tract infections in residents of nursing homes: Cluster randomised controlled trial. BMJ 2005;331:669.

8. Nicolle LE. Asymptomatic bacteriuria in the elderly. Infect Dis Clin North Am 1997;11:647-62.
The present study was the first to observe clinical outcomes as a measure of effectiveness of diagnosis and treatment for UTI in LTC.

An editorial has suggested that there is adequate equipoise to propose a randomized trial comparing delayed antibiotic therapy with immediate antibiotic therapy, or comparing hydration to antibiotic therapy (14). A further suggestion to randomly assign LTC patients to management with urine culture or no urine culture appears to be reasonable. Further research regarding clinical outcomes is needed, including the effect of urine culture testing on mortality.

A laboratory policy to provide urine culture testing services only to selected LTC patients may reduce antibiotic consumption, although if physicians treat without culture results, this reduction would not be achieved. Requiring physicians to call the laboratory to receive urine culture reports reduced inappropriate treatment for asymptomatic bacteriuria among inpatients by $36 \%$ (15), suggesting that laboratory policy interventions may influence antimicrobial use significantly. At the same time, investigations such as blood culture could be encouraged. Blood culture results have greater specificity for infection and influence therapy more directly.

AUTHOR CONTRIBUTIONS: PD: proposal, ethics, analysis, manuscript; CP: data collection, analysis; SW: data collection; BB: manuscript editing; LN: manuscript editing.

9. Nicolle LE, Bjornson J, Harding GK, MacDonell JA. Bacteriuria in elderly institutionalized men. N Engl J Med 1983;309:1420-5.

10. Abrutyn E, Mossey J, Berlin JA, et al. Does asymptomatic bacteriuria predict mortality and does antimicrobial treatment reduce mortality in elderly ambulatory women? Ann Intern Med 1994;120:827-33.

11. Loeb M, Bentley DW, Bradley S, et al. Development of minimum criteria for the initiation of antibiotics in residents of long-termcare facilities: Results of a consensus conference. Infect Control Hosp Epidemiol 2001;22:120-4.

12. Juthani-Mehta M, Quagliarello V, Perrelli E, Towle V, Van Ness PH, Tinetti M. Clinical features to identify urinary tract infection in nursing home residents: A cohort study. J Am Geriatr Soc 2009;57:963-70

13. Juthani-Mehta M, Tinetti M, Perrelli E, Towle V, Van Ness PH, Quagliarello V. Diagnostic accuracy of criteria for urinary tract infection in a cohort of nursing home residents. J Am Geriatr Soc 2007;55:1072-7.

14. Nicolle LE. Symptomatic urinary tract infection in nursing home residents. J Am Geriatr Soc 2009;57:1113-4.

15. Leis JA, Rebick GW, Daneman N, et al. Reducing antimicrobial therapy for asymptomatic bacteriuria among noncatheterized inpatients: A proof-of-concept study. Clin Infect Dis 2014;58:980-3. 


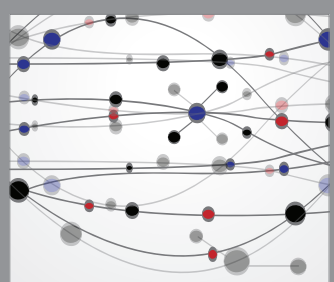

The Scientific World Journal
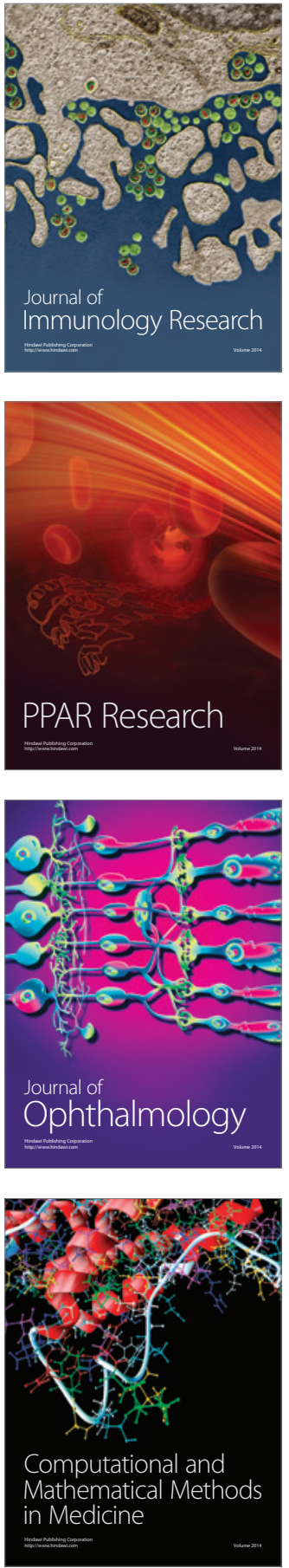

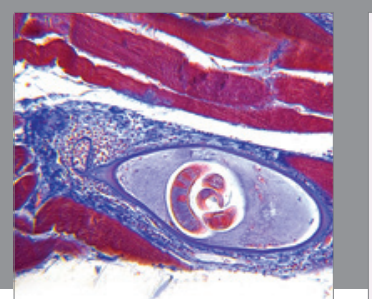

Gastroenterology Research and Practice

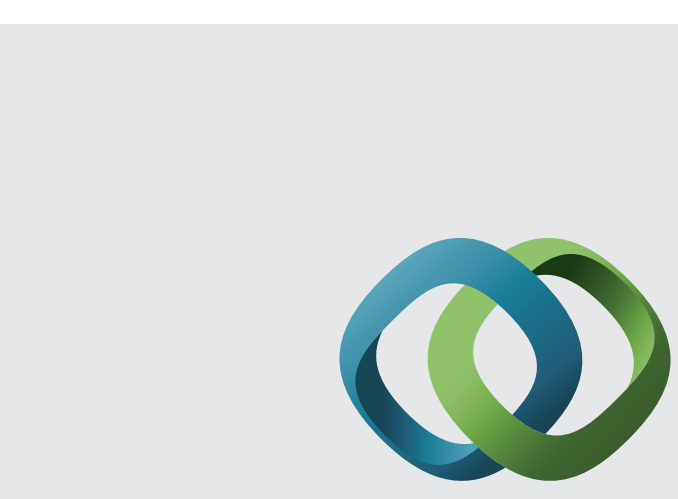

\section{Hindawi}

Submit your manuscripts at

http://www.hindawi.com
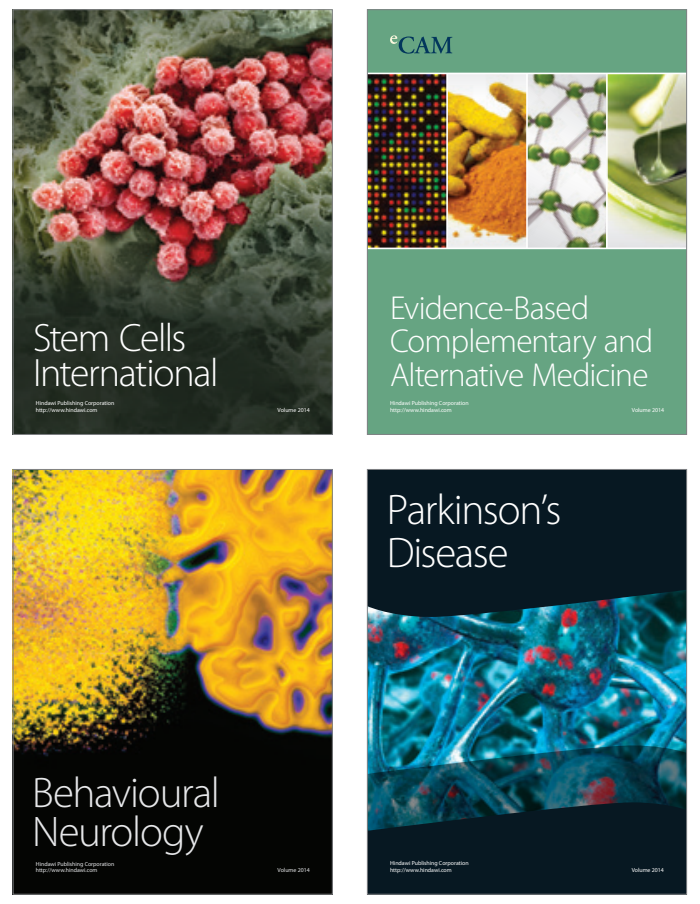
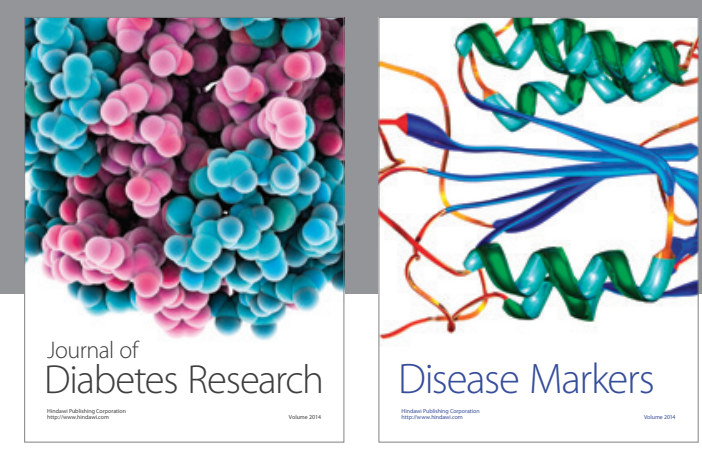

Disease Markers
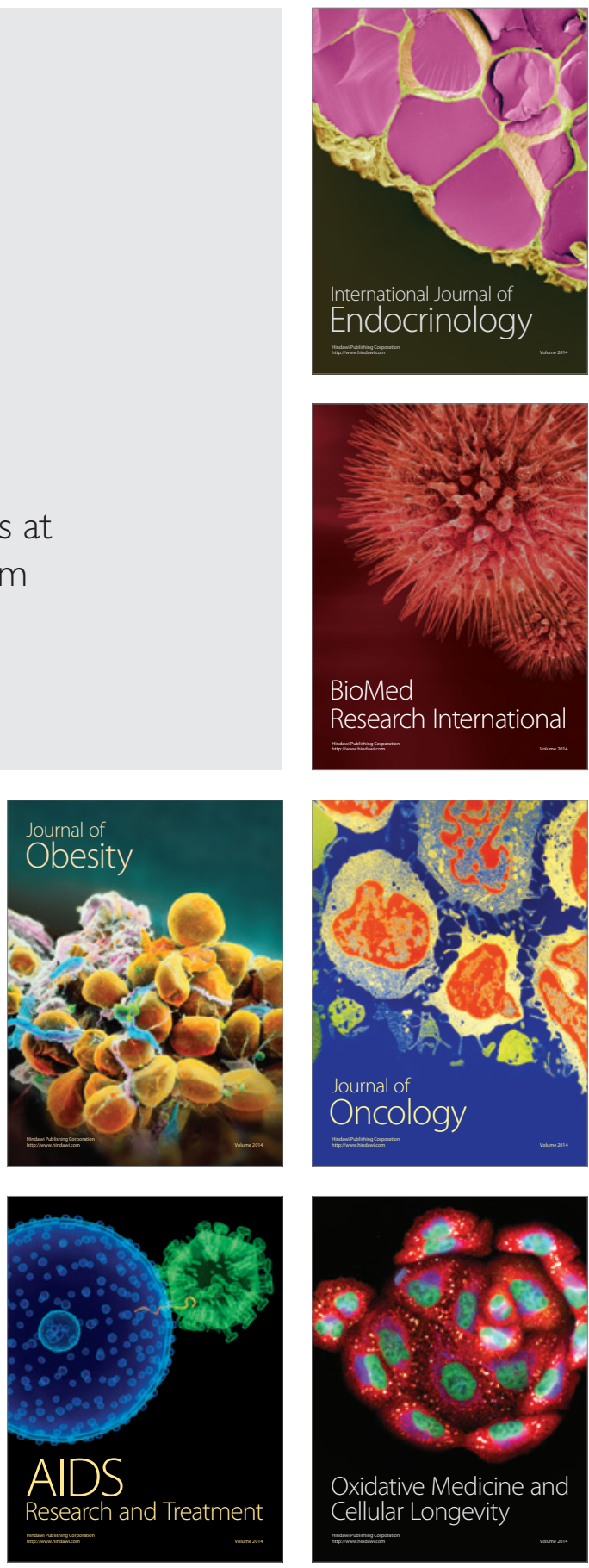